\title{
Numerical analysis of compressible turbulent helical flow in a Ranque-Hilsch vortex tube
}

\author{
R. Ricci, A. Secchiaroli, V. D’Alessandro \& S. Montelpare \\ Dipartimento di Energetica - Facoltà di Ingegneria Università \\ Politecnica delle Marche, Italy
}

\begin{abstract}
Numerical analysis of the internal flow field in a Ranque-Hilsch vortex tube (RHVT) has been conducted in order to improve understanding of its fluiddynamic behaviour. The flow field in an RHVT is compressible, turbulent and helical with a very high degree of swirl; hence its numerical simulation is a challenging task. Particular interest has been reserved for turbulence modelling, hence both RANS and LES approaches have been employed. In particular axialsymmetric RANS simulations have been conducted using RNG k- $\varepsilon$ and a linear RSM (Reynolds Stress differential Model) closure models, while full threedimensional LESs have been performed using Smagorinsky and Germano-Lilly sub-grid scales (SGS) models. Results showed, that turbulence closure models choice is a crucial issue in the prediction of the flow field in an RHVT. In fact, different simulations exhibit some differences in the description of the velocity vector components. In each simulation, flow government equations have been solved using the commercial finite volume code FLUENT'TM 6.3.26.

Flow patterns in this device have been also investigated by means of the calculation of the Helical Flow Index or normalized helicity; Power Spectral Density (PSD) of velocity magnitude has been eventually calculated showing a good agreement with K41 theory. An improved understanding of the flow field inside the RHVT can lead to a correct prediction of fluid dynamic and thermal behaviour of outlet jets, fundamental information to define cooling performance of this device.
\end{abstract}

Keywords: Ranque-Hilsch vortex tube, swirl flows, RANS, LES, turbulent spectrum. 


\section{Introduction}

Ranque-Hilsch vortex tube (RHVT) is a simple device able to split a compressed gas flow into two low-pressure flows with temperature higher and lower of the inlet gas respectively, Fig. 1. This effect, called Ranque-Hilsch effect or "thermal separation", is only due to the fluid dynamic behavior of the device. The RHVT consists of a circular tube with an inlet section, where the compressed gas flow enters tangentially through several nozzles, azimuthally arranged. The highpressure flow (by means of a very strong swirling motion) is split into two streams that flow near the internal wall of the tube, the hot one, and along the axis, the cold one (Fig. 1). These gas streams leave the device through two axial outlet sections that are located on the opposite sides of the tube (counter flow tube). Although the vortex tube is a simple device the fluid dynamic effect that produce thermal separation is extremely complex and not completely understood. Many efforts to explain this phenomenon have been made in the past, based on theoretical, numerical and experimental analysis. A complete review can be found in Eiamsa-ard and Promvonge [4]. In this work a numerical analysis of the internal flow field in an RHVT has been conducted using the computational model defined by Ricci et al. [7].

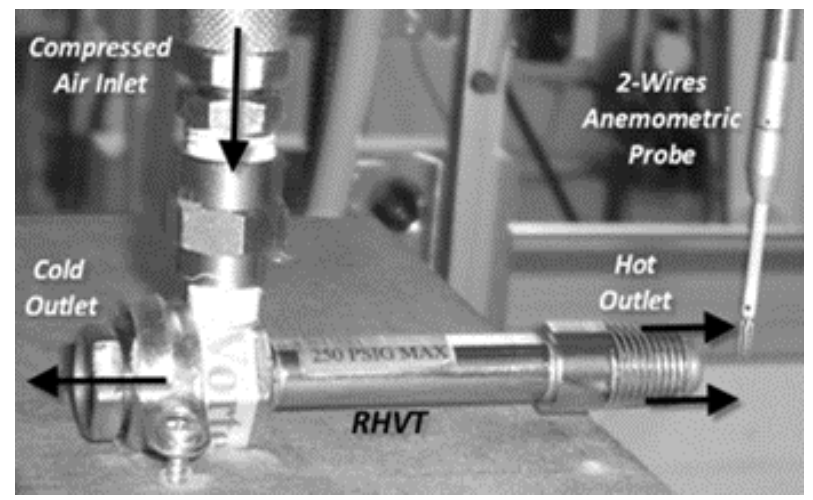

Figure 1: $\quad$ RHVT commercial model used in this work: Exair ${ }^{\circledR} 25 \mathrm{scfm}$.

\section{RHVT model description}

Computational model has been built introducing several simplifications that involve inflow and outflow sections. Inlet section has been modified following Skye et al. [1], while outlet hot section has been represented as an axial outflow (instead of radial ones reported in previous papers) closer to real geometry. Computational models are shown in figs. 2 and 3. An axial-symmetric domain has been used for RANS simulations, while three-dimensional grids have been built for LESs. Grid independence procedure, for both RANS simulations and LESs, has been performed by means of Richardson extrapolation technique (Roache [14]), ensuring a negligible influence of grid spacing on the results. Grid features used in these simulations are described in Ricci et al. [7]. 


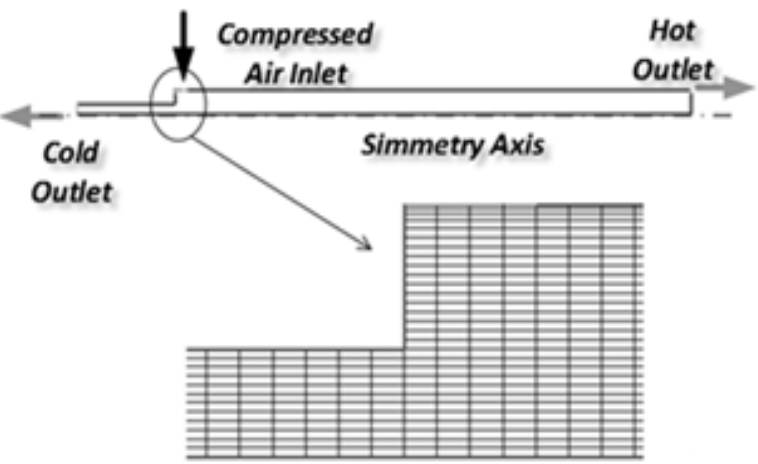

Figure 2: $\quad$ Sketch of a RANS grid.

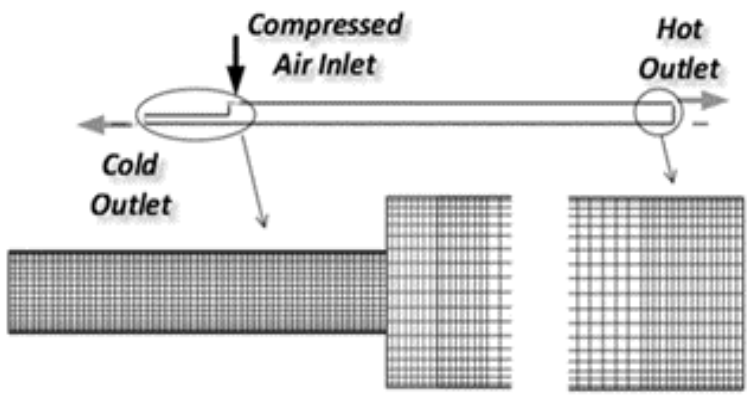

Figure 3: $\quad$ Sketch of a LES grid.

\section{Mathematical model}

The complete set of governing equations is represented by Navier-Stokes, in which gravity effects are neglected, stress tensor is related to strain rate one by constitutive relations for Newtonian fluids, and thermal flux vector is expressed by Fourier's postulate. Thermophysical properties of air supplying the device are represented by a third order polynomial function.

Boundary conditions are expressed, following Skye et al. [1] by imposing pressure, total temperature, velocity vector components and mass flow rate values at the computational inlet, in addiction to pressure values at the outlets as in table 1 . No-slip and adiabatic conditions are set at the solid bounds.

\section{Turbulence models}

The turbulent behaviour of the fluid inside the RHVT has been analyzed in this work by means RANS and LES approach. Being the medium a compressible fluid we have to take into account density and temperature fluctuations (in 
addition to velocity and pressure ones). Nevertheless in the case of compressible flows, it is useful to eliminate density turbulent fluctuations from mean motion equation using Favre's (or mass-weighted) average (for more details see Wilcox [9]).

Table 1: $\quad$ Boundary conditions.

\begin{tabular}{lc}
\hline Parameter & Value \\
Pressure Inlet $\boldsymbol{p}_{\boldsymbol{i n}}[\mathrm{Pa}]$ & 700000 \\
Hot Press. Outlet $\boldsymbol{p}_{\boldsymbol{h}}[\mathrm{Pa}]$ & 101325 \\
Cold Press. Outlet $\boldsymbol{p}_{\boldsymbol{c}}[\mathrm{Pa}]$ & 101325 \\
Inlet Total Temp. $\mathrm{T}_{\mathrm{in}}[\mathrm{K}]$ & 300 \\
Tangential Velocity $\mathrm{v}_{\boldsymbol{\theta}}[\mathrm{m} / \mathrm{s}]$ & 47.3 \\
Radial Velocity $\mathrm{v}_{\mathrm{r}}[\mathrm{m} / \mathrm{s}]$ & 9.5 \\
\hline
\end{tabular}

This approach has been used in the present work too. In this research, two turbulence models have been used for RANS equations closure: RNG k- $\varepsilon$ and a linear RSM (Wilcox [9]). In contrast to RANS approach the Large Eddy Simulation problem formulation must be unsteady and three-dimensional, so neither geometrical nor analytical simplifications are possible. Filtering operation in LESs has been performed by using Favre filter for each flow function $\boldsymbol{f}(1)$, as described in Erlebacher et al. [10].

$$
\begin{array}{r}
\widetilde{f}(\mathbf{x}, t)=\frac{\int_{\Omega \times[0, T]} \rho f\left(\mathbf{y}, t^{\prime}\right) G\left(\mathbf{x}-\mathbf{y}, t^{\prime}\right) d^{3} \mathbf{y} d t^{\prime}}{\int_{\Omega \times[0, T]} \rho\left(\mathbf{y}, t^{\prime}\right) G\left(\mathbf{x}-\mathbf{y}, t^{\prime}\right) d^{3} \mathbf{y} d t^{\prime}} \\
G(\mathbf{x}-\mathbf{y})= \begin{cases}1 / V & \mathbf{y} \in V \\
0 & \mathbf{y} \text { otherwise }\end{cases}
\end{array}
$$

Two subgrid-scales models have been used in LES: Smagorinsky [11] and Germano-Lilly (dynamic) [12]. Through finite volume discretization, a simplification of the expression of unresolved terms in LES equations can be obtained, using a filter function $G(\mathbf{x}-\mathbf{y})$ defined by (2), i.e. stating the equivalence between filter width and grid width. In this case in fact Leonard Stress Tensor and Cross Stress Tensor can be neglected.

\section{Numerical methods}

Flow government equations have been solved by means of a second order Finite Volume Method (see Jasak [13]). In RANS equations the discretization of convective terms in mass, momentum and energy conservation equations has been performed by a SOU scheme, while, discretization scheme used for $\mathrm{k}, \varepsilon$ and 
Reynolds stresses transport equations is a QUICK. In Large Eddy Simulations the discretization scheme used for convective terms is a low diffusion MUSCL while time integration has been performed with a second order accurate implicit scheme. Frictionless flux treatment has been performed by means of a Roe Flux scheme. Further information and details of all the numerical schemes used in this work can be found in the Fluent user's guide [8].

\section{Results}

By the reason of high computational cost, physical time simulated has been set equal to $20[\mu \mathrm{s}]$. Anyway the achievement of steady condition has been evaluated by monitoring time history of the integral pressure on the central section of the RHVT (fig. 4). Physical time simulated is probably large enough to allow the achievement of a steady condition for swirl velocity, axial velocity (the most important ones) and temperature as shown in figs. 5, 6 and 7.

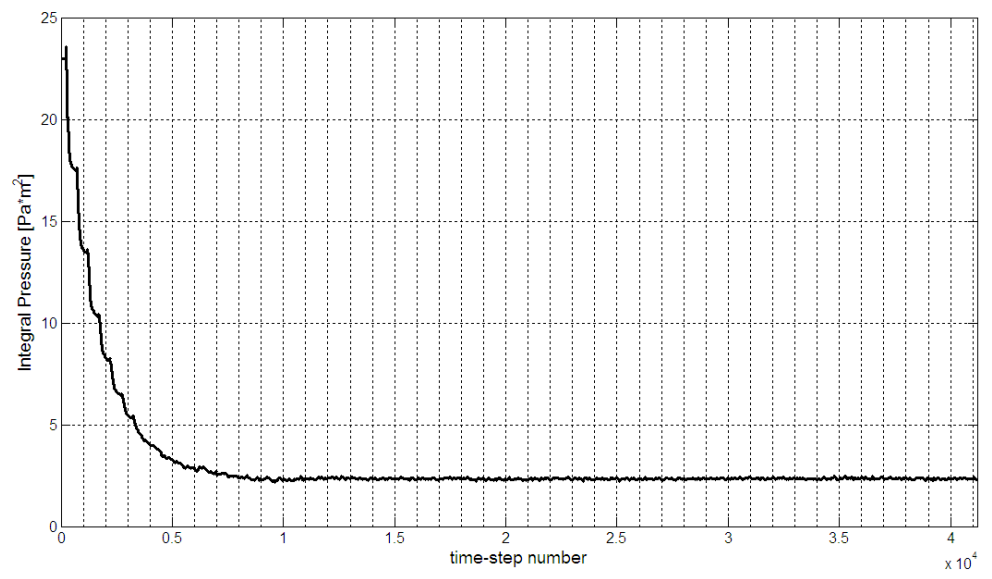

Figure 4: Integral Pressure value versus time step number (LES Smagorinsky model).

LES results, presented in this paper, have been obtained averaging instantaneous quantities on a number of time steps corresponding to the steady condition. A complete comparison between velocity profiles simulated by the different turbulent models employed in this work is reported in figs. 8 and 9 for several positions along the tube axis.

In these figures is underlined that, in high swirl conditions, the most simple turbulent model predicts velocity profiles very different from LES and RSM ones. All the mathematical models, used in this work, show that swirl velocity is the highest component, as reported in Skye et al. [1], Farouk and Farouk [2], Eiamsa-ard and Promvonge [3] and Behera et al. [5]. Anyway only LESs and RSM closure are able to predict radial profiles of swirl velocity similar to Rankine Vortex ones that are typical for these flow conditions. 


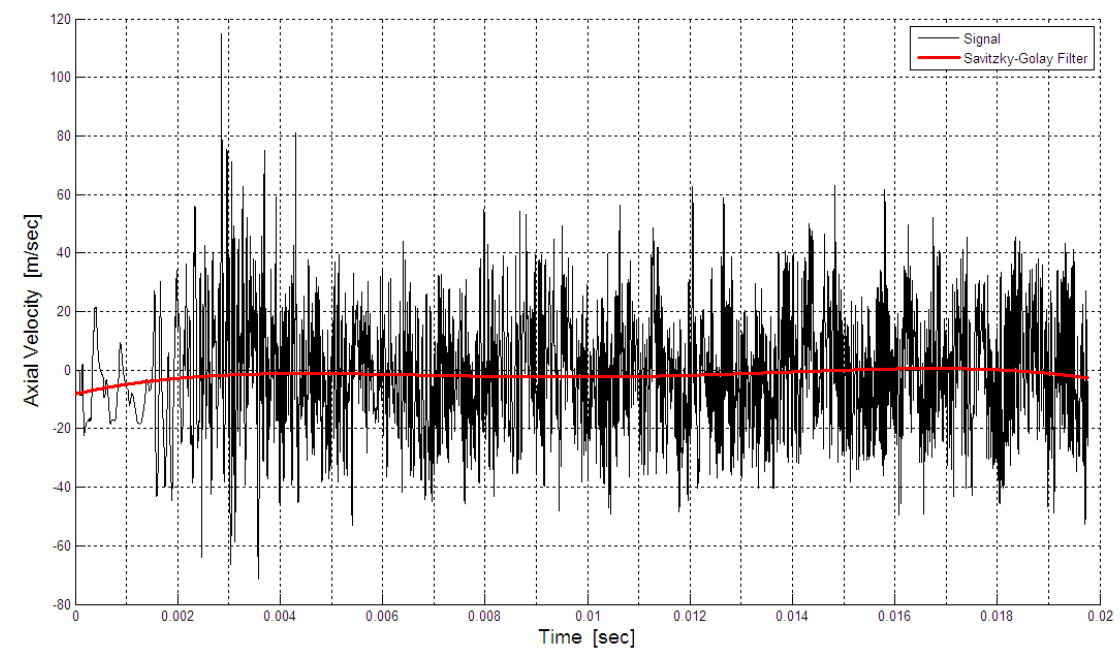

Figure 5: Axial velocity values versus time in the point at $50 \mathrm{~mm}$ from the hot exit and at a $1.75 \mathrm{~mm}$ in vertical direction from the axis (Germano-Lilly model).

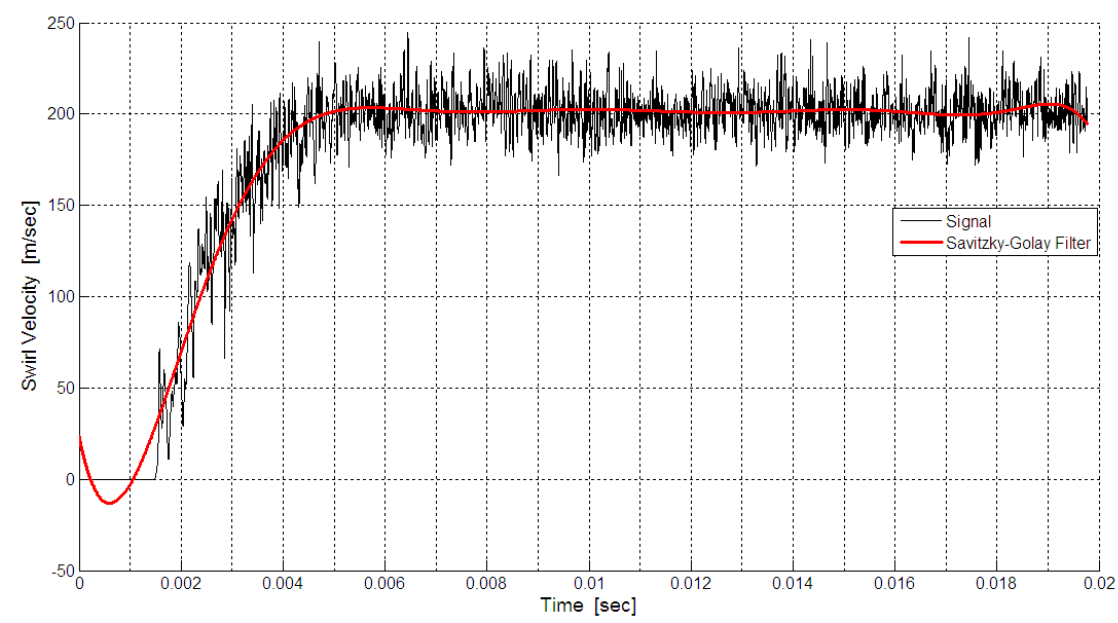

Figure 6: Swirl velocity values versus time in the point at $50 \mathrm{~mm}$ from the hot exit and at a $1.75 \mathrm{~mm}$ in vertical direction from the axis (Germano-Lilly model).

Moreover LESs and RSM approaches show a good agreement in the prediction of swirl velocity profile, nevertheless the maximum value is overestimated by the RSM approach. Except for the regions near the axis and near the wall, simulations with RNG k- $\varepsilon$ turbulence model show a swirl velocity field very similar to rigid body rotation one. These results are in agreement with a previous work (Behera et al. [5]) using the same turbulence closure, even 
though in an RHVT with different geometrical characteristics. RNG k- $\varepsilon$ model, as we expected, produced predictions with important differences respect to those obtained by RSM and LESs for the velocity field in a high swirling flows. In particular swirl velocity values are underestimated and hence axial velocity is overestimated, by this model. Axial velocity profiles show a substantial difference between RNG k- $\varepsilon$ and RSM calculations, mainly in the prediction of the maximum value. In fact, RSM closure shows a velocity decrease near the axis, while RNG $\mathrm{k}-\varepsilon$ simulations show the maximum value of axial velocity on the axis. LES results seem close to those obtained by RANS simulation with RSM and far away from results obtained by using a Boussinesque-type closure. The SGS models used in this work have not shown considerable differences in the predicted swirl velocity profiles. Anyway, the Dynamic model predicts axial velocity component values higher than Smagorinsky's ones in the central zone of the tube, while, near the wall region, Smagorinsky's model predicts higher values.

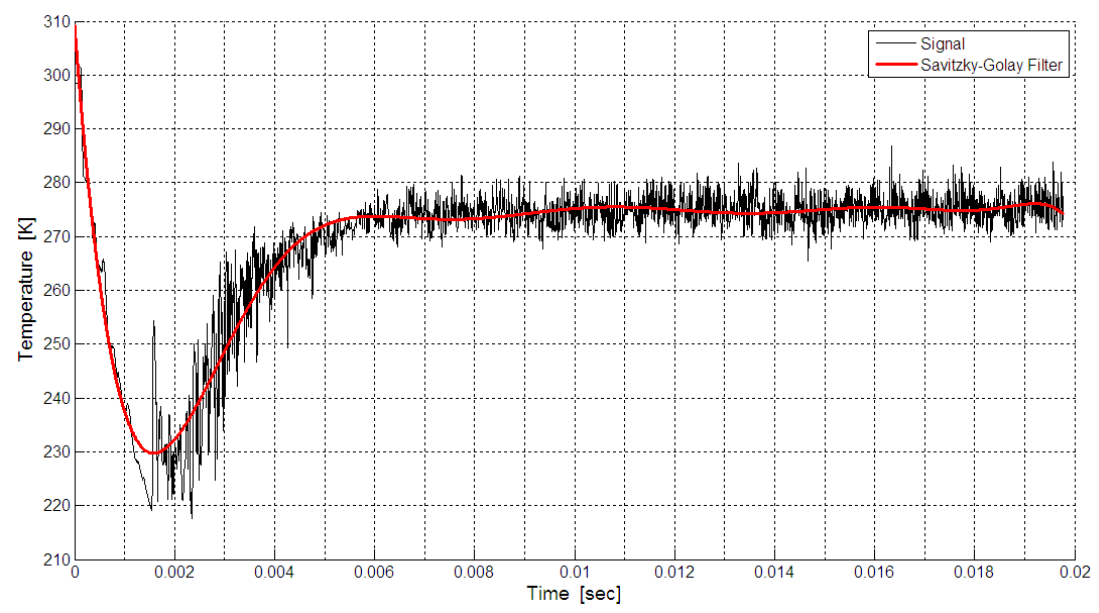

Figure 7: Temperature values versus time in the point at $50 \mathrm{~mm}$ from the hot exit and at a $1.75 \mathrm{~mm}$ in vertical direction from the axis (Germano-Lilly model).

Flow field degree of swirl is usually characterized by the swirl number $\mathrm{S}$, representing axial flux of swirl momentum divided by axial flux of axial momentum and expressed in (3), in which $\mathbf{u}$ represents the velocity vector, $w$ the swirl velocity component, $u$ the axial velocity component, $\rho$ the fluid density and $r$ the distance of the generic fluid element from the RHVT axis .

$$
S=\frac{\int_{A} \rho r w \mathbf{u} \cdot d \mathbf{A}}{R \int_{A} \rho u \mathbf{u} \cdot d \mathbf{A}}
$$




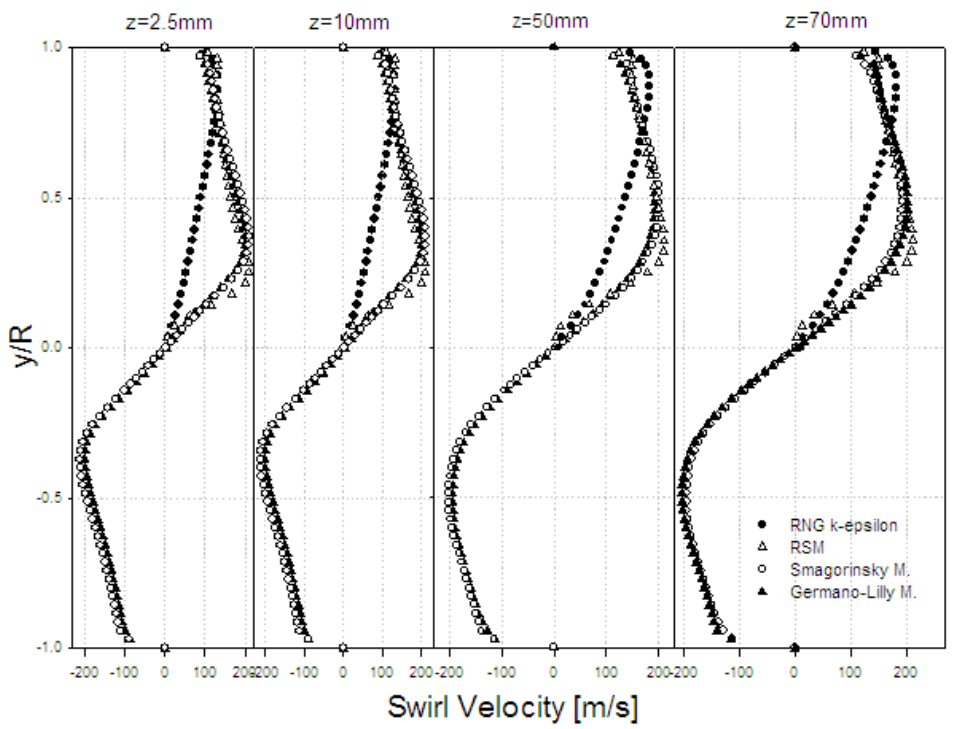

Figure 8: $\quad$ Swirl Velocity profiles obtained with all Turbulence Models used in this work at several distances form hot outlet.

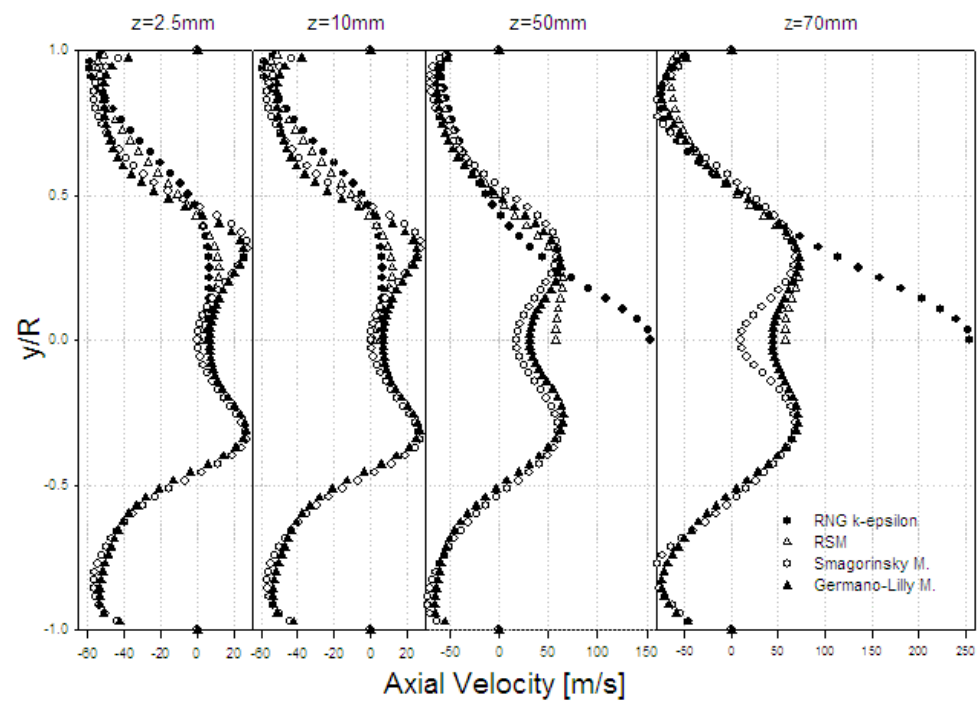

Figure 9: Axial Velocity profiles obtained with all Turbulence Models used in this work at several distances form hot outlet. 


$$
S=\frac{\sum_{f} \rho_{f} r_{f} w_{f} \mathbf{u}_{f} \cdot \mathbf{A}_{f}}{R \sum_{f} \rho_{f} u_{f} \mathbf{u}_{f} \cdot \mathbf{A}_{f}}
$$

Swirl Number has been calculated numerically by means of a second order finite volume method over LESs data on several radial planes along RHVT axis, as in (4), where subscript $f$ means that the variable is calculated on the face of the computational volume. This computation has been shown that the most intense swirl is located near the inflow section; moreover swirl velocity increase is shown close to hot outlet where axial velocity decreases. Results are reported in fig. 10 and are almost independent from subgrid-scales model used.

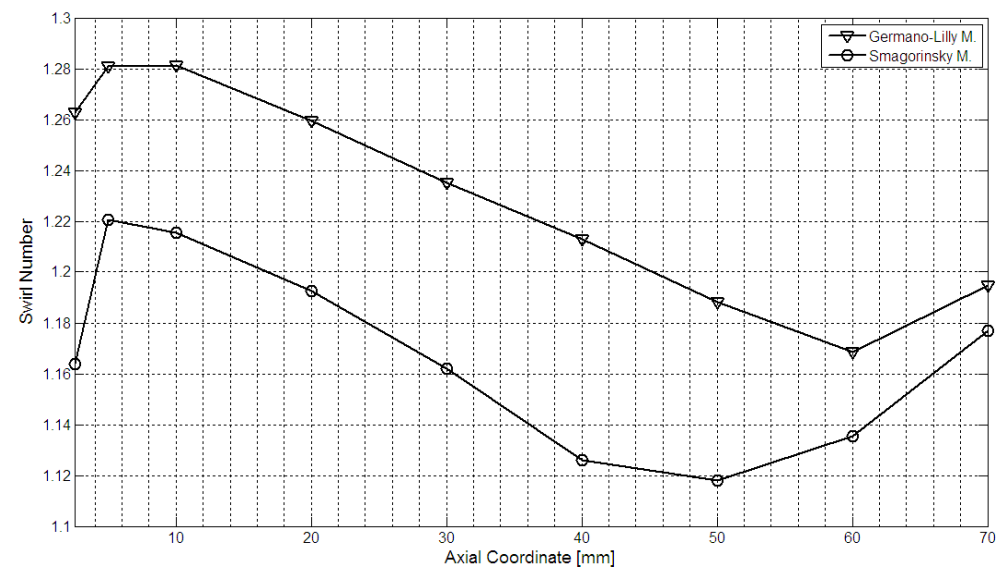

Figure 10: Swirl Number calculated on several radial planes along RHVT axis.

To improve the understanding of the instantaneous flow features the Helical Flow Index $\Psi$ (5) has been used in LES results analysis. HFI is a parameter, defined as in Morbiducci et al. [15] and varying between -1 and 1 in reason of Cauchy-Schwarz inequality. When $\Psi=1$ the flow is helical and $\Psi=0$ if the flow is purely axial or circumferential. Different HFI contours on several planes inside the tube are reported in Fig. 11.

This analysis showed an instantaneous pure helical flow near the wall (in some radial planes), an almost purely axial flow near the tube axis and a hybrid motion in the rest of the domain where $0<|\Psi|<1$.

$$
\Psi(\mathbf{x}, t)=\frac{\mathbf{u} \cdot(\nabla \times \mathbf{u})}{|\mathbf{u}||\nabla \times \mathbf{u}|}
$$




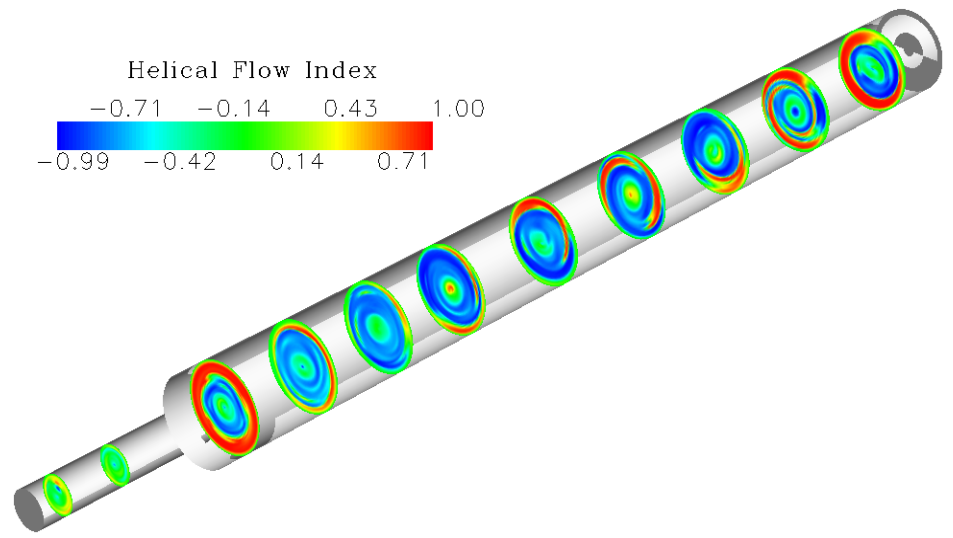

Figure 11: HFI instantaneous contours on several radial planes (Smagorinsky model).

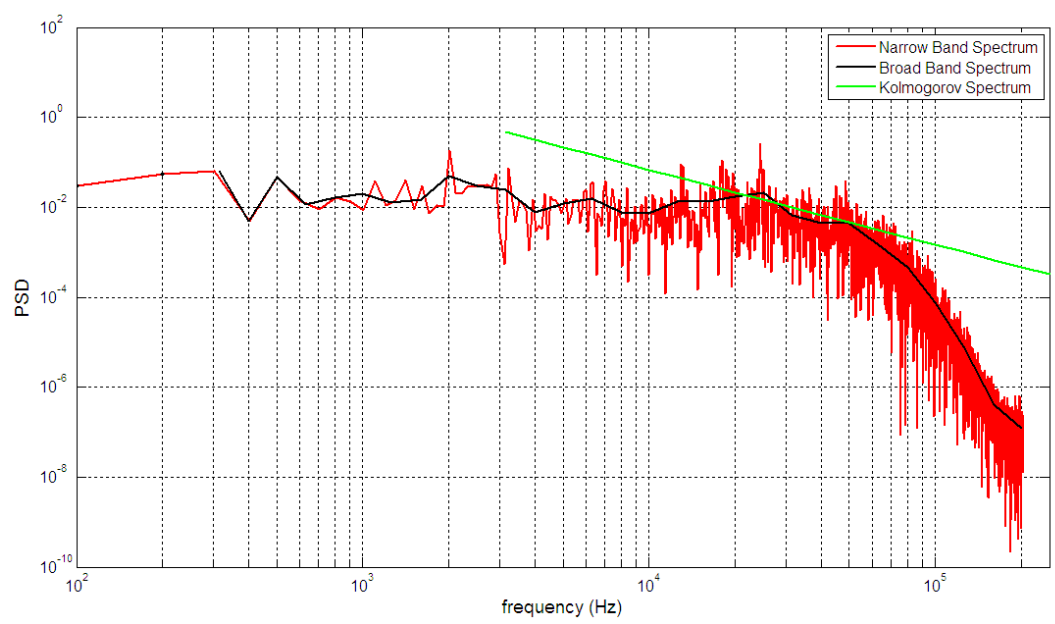

Figure 12: PSD calculated in a point by LES ( Germano-Lilly model).

A spectral analysis of the flow field using LES data has been done. Power Spectral Density has been obtained by means of a DFT algorithm. Calculations are referred to a point located on a vertical diameter at a distance of $50 \mathrm{~mm}$ from the hot exit and of $1.75 \mathrm{~mm}$ from the axis. Broad (third octave) and narrow band spectra for Smagorinsky and Germano-Lilly models data are presented in Figs. 12, 13.

\section{Conclusions}

Both RANS and LES approaches have been tested using different RANS closures and SGS models. Results showed that the flow in the tube is split in two helical 
co-axial, co-rotating streams, with different thermal features, placed near the internal wall of the tube, the hot one, and near the axis, the cold one. Flow patterns and velocity profiles show a good agreement with results reported in Skye et al. [1], Farouk and Farouk [2], Eiamsa-ard and Promvonge [3] and Behera et al. [5].

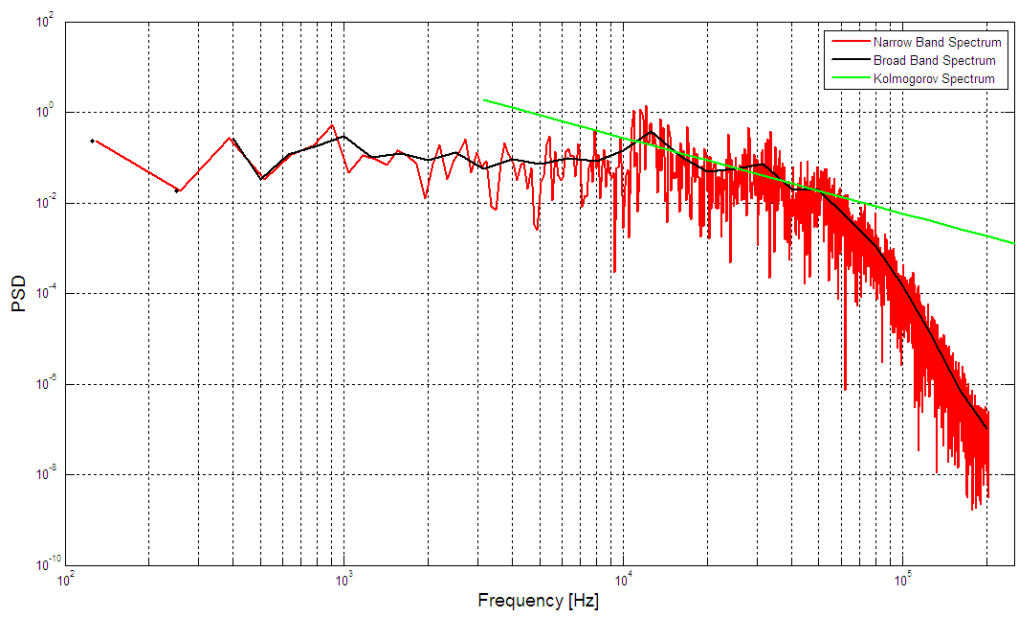

Figure 13: $\quad$ PSD calculated in a point by LES (Smagorinsky model).

In LES, with Germano-Lilly model, some differences have been shown in the prediction of the axial velocity field while swirl velocity prediction seemed to be unaffected by a SGS model variation. Due to high computation time, it was not possible to increase physical time simulated, hence for the radial velocity, which has a slower dynamic behaviour, a stationary condition has not probably been reached.

Swirl Number calculation showed that the most intense swirl is located near inflow; moreover a swirl velocity increase is shown close to hot outlet where axial velocity decreases. Preliminary results of spectral analysis from LES data showed a good description of the inertial sub-range.

\section{References}

[1] Skye, H.M., Nellis, G.F. \& Klein, S.A., Comparison of CFD analysis to empirical data in a commercial vortex tube. Int. J. of Refrigeration 29, pp. 71-80, 2006.

[2] Farouk, T. \& Farouk, B., Large eddy simulations of the flow field and temperature separation in the Ranque-Hilsch vortex tube. Int. J. of Heat and Mass Transfer, 50, pp. 4724-4735, 2007.

[3] Eiamsa-ard, S., \& Promvonge, P., Numerical investigation of the thermal separation in a Ranque-Hilsch vortex tube. Int. J. of Heat and Mass Transfer, 50 (5-6), pp. 821-832, 2007. 
[4] Eiamsa-ard, S., \& Promvonge, P., Review of Ranque Hilsch effect in vortex tubes, Renewable and Sustainable Energy Reviews 12, pp. 18221842, 2008.

[5] Behera, U., Paul, P.J., Kasthurirengan S., Karunanithi, R., Ram, S.N. \& Dinesh, K., CFD analysis and experimental investigations towards optimizing the parameters of Ranque-Hilsch vortex tube. Int. J. of Heat and Mass Transfer 48, pp. 1961-1973, 2005.

[6] Ricci, R., Secchiaroli, A., Montelpare, S. \& D’Alessandro, V., Fluid Dynamics Analysis of Ranque-Hilsch Vortex Tube. Rivista del Nuovo Cimento (to appear), 2009.

[7] Ricci, R., D’Alessandro, V., Secchiaroli, A., Montelpare, S. \& Mazzieri, M., Raffreddamento di Componenti Elettronici di Potenza Mediante Dispositivi ad Effetto Ranque-Hilsch: Simulazione Numerica del Flusso Interno ed Esterno. Proceedings of 26th UIT National Heat Transfer Conference, Ed. ETS, pp. 527-532, 2008.

[8] Fluent Inc. "FLUENT 6.3.26 Users' Guide" 2006.

[9] Wilcox, D.C., Turbulence Modelling for CFD, DCW Industries, California, USA, 1994.

[10] Erlebacher, M., Hussaini, Y., Speziale \& C. G., Zang, T. A., Toward the Large-Eddy Simulation of Compressible Turbulent Flows. J. of Fluid Mechanics 238, pp. 155-185, 1992.

[11] Smagorinsky, J., General circulation experiments with primitive equations, I. The basic experiment. Month. Weather Rev, 91, pp. 99-164, 1963.

[12] Germano M., Piomelli U., Moin P. \& Cabot W.H. A dynamic SGS eddyviscosity model, Physics of Fluids A3(7), pp. 1760-1765, 1991.

[13] Jasak, H., Error analysis and estimation for the Finite Volume method with applications to fluid flows. PhD Thesis Imperial College, London, UK, 1996.

[14] Roache, P.J., Verification and Validation in Computational Science and Engineering. Hermosa Publishers, 1998.

[15] Morbiducci, U., Del Gaudio, C., D’Avenio, G., Calducci, A. \& Barbario, V., A mathematical description of blood spiral flow in vessels: application to a numerical study of flow in arterial bending. J. of Biomechanics 38 (7), pp. 1375-1386, 2005. 